\title{
La universalidad de los prototipos semánticos en el léxico disponible de español
}

Palabras clave: prototipos semánticos, disponibilidad léxica, teoría de prototipos, léxico más disponible, español como lengua extranjera

\section{Introducción}

En todas las civilizaciones conocidas, incluso algunas que han vivido aisladas del resto del mundo, a pesar de las muchas diferencias, han desarrollado una lengua más o menos sofisticada que sirve exactamente a los mismos propósitos en cada una de ellas: informar, pedir información, amar, regañar, amenazar, etc. Ninguna lengua es completamente diferente, todas tienen particularidades y universalidades que son cara y cruz de cada lengua. Se parte aquí de la idea de que las lenguas diferentes tienen mucho en común y de que son muchos más los factores que unifican a los hablantes que los que los distancian.

A pesar de la enorme diversidad léxica y conceptual que puede hallarse en las lenguas del mundo se puede constatar que en todas las lenguas, para todos los pueblos y en todas las épocas, la palabra cumple básicamente funciones semejantes ya que surge de las mismas necesidades de la condición humana, es decir, de la misma biología y psicología humanas, del mismo reflejo básico del entorno medioambiental y de las mismas necesidades comunicacionales. Por esta razón no es de extrañar que exista mucho en común en el léxico de las diferentes lenguas del mundo y estas semejanzas, lo mismo que las diferencias, pueden ser racionalizadas y sistematizadas. (Luque Durán, 2004: 16) 
Asimismo podemos conectar estas ideas con el concepto del lenguaje como un instinto, propuesto por Pinker (1995, 2010). Según este psicólogo existe un instinto en la especie humana que le hace desarrollar un lenguaje oral diferenciado de todas las formas de comunicación animal, y, como instinto, es innato y está codificado en nuestros genes, siendo el cerebro el órgano físico dedicado a ello. Pinker ( $I b i d$.) asegura que la habilidad del lenguaje es tan natural como lo es la destreza que demuestran las arañas en tejer sus telas. Si el lenguaje es tan natural y universal, ¿pensamos entonces en nuestra lengua materna o extranjera o acaso «nuestros pensamientos se formulan por mediación de un vehículo silencioso del cerebro, una especie de lenguaje del pensamiento o idioma mentalés, para luego revestirlos de palabras cuando se hace preciso comunicárselos a un interlocutor?’ (Pinker, 1995: 58)

El presente trabajo tiene como objetivo investigar la concepción del lenguaje como objeto netamente natural y universal. A tal fin se ofrecen los argumentos que se encuentran en las investigaciones de las primeras y más frecuentes asociaciones a ciertos estímulos. En otras palabras, se trata de reconocer algunos universalismos en el campo de los prototipos semánticos. Nuestra principal hipótesis es que las primeras asociaciones de léxicos disponibles de diferentes culturas y lenguas permiten ser catalogadas como voces prototípicas o elementos nucleares del estímulo. Por lo tanto se presentan y comparan las palabras más disponibles de varias investigaciones de léxico disponible para extraer algunas conclusiones sobre los prototipos semánticos y así contribuir al desarrollo del campo de la psicolingüística en el sentido del lenguaje como un instrumento universal. A partir de los resultados del léxico disponible, obtenidos con fluencia semántica y a través de una fórmula matemática en la que se conjugan la frecuencia y el orden de activación de la palabra, se comparan las unidades léxicas más disponibles en español lengua materna (ELM) y español lengua extranjera (ELE).

Se trata de constatar que la disponibilidad léxica nos ayuda a conocer la organización léxica de la memoria semántica y el lexicón mental, hecho que viene avalado por los estudios de psicología cognitiva sobre las asociaciones mentales. Como el léxico más disponible se obtiene con la fluencia semántica, es decir, con las asociaciones abiertas según ciertos campos semánticos, se encuentra en esto un paralelismo con la teoría de prototipos. Se trata de responder a la pregunta de si la disponibilidad léxica en español LM de los jóvenes españoles y latinoamericanos ha dado los mismos prototipos que la disponibilidad léxica en español LE de los jóvenes eslovenos, finlandeses, turcos, chinos 
y de algunas otras nacionalidades. Para estudiar el léxico disponible se recurre a las pruebas o encuestas asociativas a partir de ciertas categorías semánticas (o también llamadas centros de interés) que funcionan como estímulos verbales. A partir de estos estímulos los informantes escriben las palabras más disponibles que acuden de manera más rápida y fácil a la mente. Para cada campo semántico disponen de dos minutos para escribir las asociaciones. Con una fórmula matemática (López Chávez-Strassburger, 1991 en López Morales, 1999: 18) se calcula el índice de disponibilidad que une criterios de la frecuencia y la posición de cada palabra.

\section{La teoría de prototipos}

La teoría de prototipos es una teoría desarrollada en el marco de la psicología cognitiva y la lingüística cognitiva que pretende ofrecer un modelo de categorización, alternativo al modelo tradicional basado en la lógica aristotélica. En cada categoría, como señala Martos Ramos (2010: 248), el prototipo es el elemento más importante y relevante desde un punto de vista semántico y cognitivo: además, contiene numerosos rasgos que favorecen la comunicación y la comprensión entre hablantes. El mismo autor (Ibid.), apoyándose en dos investigadores muy importantes del campo, concluye que «no es por casualidad que se trate del elemento de la categoría que menos esfuerzo cognitivo requiere y que al mismo tiempo mayor información transmite (Rosch, 1973; Lakoff, 1987)».

En la versión estándar, formulada por Rosch y su grupo al inicio de los setenta, el prototipo es considerado «como el ejemplar más idóneo e incluso el mejor caso, el mejor representante o caso central de una categoría» (Kleiber, 1995: 47), para pasar posteriormente a definirse como una dimensión colectiva: «el ejemplar idóneo comúnmente asociado a una categoría» (Kleiber, 1995: 49). La teoría de los prototipos rompe con la concepción clásica, aristotélica, de la categorización y propone una nueva teoría de la categorización, la cual no considera que la existencia de propiedades comunes compartidas por todos los miembros sea condición necesaria para el establecimiento de una categoría. Podemos decir que mientras que para el modelo clásico todos los miembros de una categoría lo son por cumplir iguales condiciones (condiciones necesarias y suficientes), es decir, todos los pájaros son igual de pájaros una vez han sido incluidos en esa categoría, para la semántica de los prototipos unos elementos de la categoría son más pájaros que otros. 
Para Rosch (1975 en Kleiber, 1995: 56), el prototipo actúa como «punto de referente cognitivo» (cognitive reference point) para los procesos de clasificación de los elementos de nuestra experiencia. Los experimentos de verificación de los principios derivados del modelo han permitido llegar a las siguientes conclusiones:

a) Los miembros prototípicos son categorizados más rápidamente que los miembros que no son prototípicos.

b) Los miembros prototípicos son los que primero aprenden los niños.

c) Los prototipos sirven de punto de referencia cognitiva.

d) Generalmente, cuando lo que se pide es la enumeración de los miembros de una categoría, los prototipos aparecen mencionados en primer lugar. (Kleiber, 1995: 57)

Kleiber (1995: 80) señala que siguiendo los estudios de Berlin sobre las taxonomías populares, Rosch y su equipo establecen la existencia de una dimensión horizontal, es decir, una organización intercategorial jerárquica. Su propuesta reduce de cinco (Berlin) a tres el número de niveles dentro de las categorías: 1) nivel supraordinado (i.e. animal, fruta, mueble); 2 ) nivel de base (i.e. perro, manzana, silla); 3) nivel subordinado (i.e. labrador, golden, silla plegable). A1 establecer esta jerarquía, se determinaba un nivel privilegiado dentro de la categoría, el nivel de base. Kleiber (1995: 84) describe así las características del nivel de base, señalando que son prioritarias en tres planos:

- plano perceptivo, a través de la sensación de una forma global similar, como la representación por medio de una simple imagen mental de toda la categoría y de una identificación rápida;

- plano funcional, por medio de un programa motor general similar;

- plano comunicativo, en el empleo de palabras que, por una parte son las más cortas, las más comúnmente empleadas y utilizadas en los contextos neutros, y por otra, son las que primero aprenden los niños y las primeras que entran en el léxico de una lengua.

Aitchison (1994: 68-70), dentro de la teoría de los prototipos, presenta varias teorías sobre la tipicidad. En una de ellas afirma que un gorrión es más pájaro que un pingüino porque los prototipos dependen de la experiencia. Cuantas más veces vemos unos pájaros con las mismas características más probable es que las unamos en una categoría: así, en Europa es más probable que el prototipo sea gorrión que un emú. Su teoría del modelo mental es una combinación que creamos a partir de la experiencia, la observación, la influencia cultural, 
la memoria y la imaginación. Los modelos mentales presentan hipótesis que tenemos del mundo y se recopilan cuando los necesitamos. Algunos de los modelos son muy fijos (i.e. coche como prototipo de vehículo) mientras que otros son más flexibles.

Una de las objeciones más comunes a la teoría de prototipos es la falta de características propias de muchas clases y cosas, lo que hace las categorías difusas (fuzziness; Taylor, 1995: 70). Para paliar este problema se han introducido los conceptos de categorías populares ${ }^{1}$ y categorías profesionales (folk y expert categories, respectivamente; Taylor, 1995: 68), que ayudan a poner vallas (bedges; Lakoff, 1972 en Taylor, 1995: 75-80), es decir, a delimitar pragmáticamente los elementos que parecen pertenecer a dos categorías simultáneamente: por ejemplo, un murciélago no es un pájaro stricto sensu, porque es un mamífero (categoría profesional), pero en una opinión popular o infantil puede ser considerado un pájaro por poder volar (categoría popular).

Una versión posterior de la teoría de prototipos de Lakoff (1987) recoge los estudios de Wittgenstein (en Taylor, 1995: 38-40) y su concepto de semejanzas de familia, es decir, de una serie de conceptos que se parecen, así como acontece entre miembros de la misma familia (algunos se parecen más, otros menos) en su nuevo Modelo Cognitivo Idealizado (Idealized Cognitive Model). En esta nueva concepción de la teoría de prototipos, los elementos con muy pocas características en común son puestos en correlación gracias a categorías radiales, debidas a asociaciones mentales periféricas como las que pueden ocurrir entre un pájaro y un avión o con cualquier objeto capaz de volar.

Resumiendo las líneas anteriores, la organización de categorías a partir de prototipos de Rosch (en Kleiber, 1995) no es la única hipótesis pero es uno de los puntos de partida básicos sobre categorización, entendida como la organización de los conceptos sobre la base de la noción de prototipo, y, por lo tanto, nos va a servir como punto de referencia en nuestro análisis de los prototipos en el léxico disponible.

\section{La disponibilidad léxica y los prototipos}

Las encuestas de disponibilidad léxica utilizan el método de la fluencia semántica en la que se da al informante el nombre de una categoría semántica (ropa, animales, etc.) y tiene que escribir en un tiempo definido todas aquellas palabras que pertenezcan a esa categoría. En la mayoría de las encuestas se ofrecen

1 «Our everyday folk theory of what a category is» (Lakoff, 1987: 5) en Taylor (1995: 75). 
16 categorías o campos de interés con el tiempo limitado de dos minutos para cada categoría. A través de los cálculos se obtiene de las listas individuales una lista colectiva según la frecuencia y el orden de la asociación. Estos inventarios nos sirven también como diagnóstico para conocer las palabras altamente disponibles o prototípicas de cada campo semántico.

Hernández Muñoz, Izura y Ellis (2006 en Hernández Muñoz et al., 2014) recopilaron las medidas de seis factores que habían mostrado su influencia en las cien palabras más disponibles de varias categorías: familiaridad con el concepto, tipicidad, imaginabilidad, edad de adquisición, frecuencia y longitud de la palabra. Los análisis de regresión múltiple indicaron que solo tres de estos factores predecían la disponibilidad: la tipicidad (tal y como sugería la teoría de los prototipos), la familiaridad con el concepto y la edad de adquisición. Por tanto, por ejemplo, perro y gato son las palabras más disponibles para la mayor parte de los hablantes de castellano porque (1) funcionan como prototipos de animales, (2) de forma habitual entramos en contacto con el concepto al que representan y (3) las aprendimos a edad muy temprana. (Hernández Muñoz e Izura, 2010: 2)

Las categorías semánticas que mayoritariamente se usan como palabra estímulo en las investigaciones de léxico disponible son del nivel supraordinado, según la organización intercategorial jerárquica de Rosch (Kleiber, 1995: 80); en consecuencia, la mayoría de las palabras con el índice de disponibilidad más alto pertenece al grupo del nivel de base según la versión estándar de la semántica de los prototipos, ya que, entre otras características citadas en el capítulo 2, «el nivel de base es considerado como aquel en el que los individuos identifican más rápidamente a los miembros de las categorías» (Kleiber, 1995: 82) y porque «la mayor parte de nuestro conocimiento se encuentra almacenado en este nivel» (Kleiber, 1995: 84).

En el cotejo que se ofrece a continuación se demostrará si las palabras más disponibles pertenecen a esta categoría de nivel de base y si se trata de palabras que destacan por su tipicidad, familiaridad con el concepto y si se trata de palabras que aprendemos a una edad temprana en la lengua materna.

\section{El cotejo de los prototipos}

\subsection{Metodología}

A continuación se presentan las palabras más disponibles de varios inventarios de léxico disponible. De todas las sintopías comparadas se han escogido las 
primeras tres palabras ${ }^{2}$ de la lista en el caso de que las investigaciones hayan incluido las mismas categorías semánticas. Se añaden los índices de disponibilidad (ID), que aclaran mejor si cierta palabra fue escrita por muchos informantes como una de las primeras asociaciones. El índice de disponibilidad 1 significaría que todos los informantes han escrito la palabra como la primera asociación. Si el índice de cierta palabra es 0,7, podemos hablar más fielmente de prototipo que si el índice fuese 0,4 . Los campos cerrados o compactos con el índice de cohesión más alto, es decir, donde los informantes muestran asociaciones más comunes, ofrecen más delimitación de elementos prototípicos. Por lo tanto, no se ha escogido como el único criterio el índice de disponibilidad, ya que varía la capacidad asociativa según las denominaciones más o menos concretas de las categorías semánticas (i.e. El campo permite multitud de opciones en comparación con Partes del cuerpo).

Para comparar los prototipos del léxico disponible en ELE y ELM se han escogido nueve categorías semánticas comunes: Partes del cuerpo, La ropa, La casa, Alimentos y bebidas, La ciudad, El campo, Medios de transporte, Animales, Profesiones y oficios. Para presentar las voces prototípicas en ELM se han escogido los léxicos disponibles de Puerto Rico (López Morales, 1999), Asturias (Carcedo González, 2001), Cádiz (González Martínez, 2002), Valencia (Gómez Molina, R. J. y Gómez Devís, M.a B., 2004) y Melilla (Fernández Smith et al., 2008). Los léxicos disponibles en ELE presentan siete listados diferentes: los prototipos de los jóvenes preuniversitarios y universitarios de Eslovenia (Šifrar Kalan, 2011), los preuniversitarios de Finlandia (Carcedo González, 2000), los estudiantes extranjeros en los cursos de ELE en Salamanca (Samper Hernández, 2002), los adultos extranjeros en los cursos de ELE en Madrid³ (López Rivero, 2008), los estudiantes preuniversitarios turcos (González Fernández, 2013) y los estudiantes universitarios sinohablantes (Hidalgo Gallardo ${ }^{4}$.

2 Gómez Molina, J. R. y Gómez Devís, M. ${ }^{a}$ B. (2004: 282) afirman que «la estabilidad conceptual manifestada en los primeros doce vocablos, aproximadamente, de cada centro de interés permite catalogarlos como voces prototípicas o elementos nucleares del estímulo; posteriormente, se desarrollan diferentes asociaciones semánticas según subgrupos sociales $[\ldots] \gg$

3 La investigación entre los adultos extranjeros en los cursos de ELE en Madrid se llevó a cabo en seis centros de interés: Partes del cuerpo, La ropa, Alimentos y bebidas, La cocina y sus utensilios, Juegos y distracciones, La ciudad. Por lo tanto no se dispone de vocablos para los campos siguientes, que se comparan en este estudio: La casa, El campo, Medios de transporte, Animales.

4 Los datos, que el autor me ha suministrado amablemente, forman parte de una tesis doctoral en preparación sobre «El léxico disponible en estudiantes sinohablantes de ELE». 
La muestra de los léxicos disponibles en ELM consta de alrededor de 400 informantes preuniversitarios, mientras que varía en ELE: hay 100 informantes preuniversitarios y 100 universitarios de Eslovenia, 150 preuniversitaros de Finlandia, 45 informantes de los cursos de ELE en la Universidad de Salamanca, 43 informantes adultos de los cursos de ELE de Madrid, 78 informantes del último año de escuela secundaria de Turquía y 440 estudiantes universitarios de China. Ya que nuestro objetivo principal es investigar la prototipicidad $y$, con esto, la universalidad del lenguaje en general, no hemos tomado en cuenta las variables que suelen ser el objeto de estudio en este campo (el nivel del conocimiento de LE, la edad, el sexo, etc.).

\subsection{Resultados}

A continuación se presentan los cuadros por campos semánticos, separadamente para los cinco listados de ELM y siete de ELE. Las tablas incluyen las primeras tres palabras más disponibles con su índice de disponibilidad. En los cuadros de ELE se ha añadido también el dato de si los informantes son estudiantes de los dos últimos años de escuela secundaria o si se trata de estudiantes universitarios, a pesar de no incluir este dato como una variable de este estudio.

\subsubsection{Partes del cuerpo}

En el primer campo presentado en ELM, Partes del cuerpo, prevalece la palabra ojo como la más frecuente en todos los informantes y como la primera asociación para muchos informantes. Las otras palabras más disponibles son cabeza, brazo y nariz. El índice de disponibilidad (ID) en la palabra más disponible es alrededor de o,6 en ELM y un poco más alto en promedio en ELE, alrededor de 0,7 , pero en todos los ejemplos (menos en la tercera palabra más disponible en Turquía) es superior a 0,5 . Las palabras más disponibles que prevalecen en ELE son cabeza, ojo y mano, lo que muestra mucha convergencia entre los resultados de ELM y ELE.

\begin{tabular}{|l|l|l|l|l|}
\hline PUERTO RICO & ASTURIAS & CÁDIZ & VALENCIA & MELILLA \\
\hline ojo 0,61 & ojo 0,68 & cabeza 0,71 & ojo 0,72 & ojo 0,64 \\
\hline brazo 0,59 & cabeza 0,64 & ojo 0,61 & cabeza 0,59 & cabeza 0,61 \\
\hline cabeza 0,59 & brazo 0,61 & brazo 0,60 & nariz 0,59 & nariz 0,57 \\
\hline
\end{tabular}

Cuadro 1: Las palabras más disponibles de Partes del cuerpo en ELM 


\begin{tabular}{|l|l|l|l|l|l|l|}
\hline $\begin{array}{l}\text { ESLOVE- } \\
\text { NIA (pre- } \\
\text { universita- } \\
\text { rios) }\end{array}$ & $\begin{array}{l}\text { ESLO- } \\
\text { VENIA } \\
\text { (universi- } \\
\text { tarios) }\end{array}$ & $\begin{array}{l}\text { FINLAN- } \\
\text { DIA (pre- } \\
\text { universita- } \\
\text { rios) }\end{array}$ & $\begin{array}{l}\text { ESPAÑA/ } \\
\text { SALA- } \\
\text { MANCA }\end{array}$ & $\begin{array}{l}\text { ESPAÑA/ } \\
\text { MADRID }\end{array}$ & $\begin{array}{l}\text { TURQUÍA } \\
\text { (preuni- } \\
\text { versita- } \\
\text { rios) }\end{array}$ & $\begin{array}{l}\text { CHINA } \\
\text { (universi- } \\
\text { tarios) }\end{array}$ \\
\hline $\begin{array}{l}\text { cabeza } \\
0,76\end{array}$ & $\begin{array}{l}\text { cabeza } \\
0,76\end{array}$ & $\begin{array}{l}\text { mano } \\
0,69\end{array}$ & $\begin{array}{l}\text { ojo } \\
0,68\end{array}$ & $\begin{array}{l}\text { cabeza } \\
0,72\end{array}$ & $\begin{array}{l}\text { ojo } \\
0,61\end{array}$ & $\begin{array}{l}\text { ojo } \\
0,86\end{array}$ \\
\hline $\begin{array}{l}\text { mano } \\
0,75\end{array}$ & ojo & cabeza & cabeza & mano & mano & mano \\
\hline $\begin{array}{l}\text { ojo } \\
0,64\end{array}$ & 0,65 & 0,64 & 0,65 & 0,71 & 0,55 & 0,75 \\
\hline
\end{tabular}

Cuadro 2: Las palabras más disponibles de Partes del cuerpo en ELE

\subsubsection{La ropa}

Pantalón y camisa son definitivamente los prototipos de La ropa en todas las listas comparadas. El ID de estas dos palabras en ELM es superior a o,6, mientras que en ELE pantalón es superior a o,6 en cinco de los siete grupos y camisa en cuatro. Solo en el léxico disponible finlandés camisa no se encuentra entre las tres palabras más disponibles.

\begin{tabular}{|l|l|l|l|l|}
\hline PUERTO RICO & ASTURIAS & CÁDIZ & VALENCIA & MELILLA \\
\hline pantalón 0,83 & pantalón 0,83 & pantalón 0,72 & camisa 0,65 & pantalón 0,70 \\
\hline camisa 0,70 & camisa 0,69 & camisa 0,72 & pantalón 0,63 & camiseta 0,65 \\
\hline medias 0,52 & camiseta 0,66 & jersey 0,62 & calcetín 0,56 & camisa 0,60 \\
\hline
\end{tabular}

Cuadro 3: Las palabras más disponibles de La ropa en ELM

\begin{tabular}{|l|l|l|l|l|l|l|}
\hline $\begin{array}{l}\text { ESLOVE- } \\
\text { NIA (pre- } \\
\text { universita- } \\
\text { rios) }\end{array}$ & $\begin{array}{l}\text { ESLO- } \\
\text { VENIA } \\
\text { (universi- } \\
\text { tarios) }\end{array}$ & $\begin{array}{l}\text { FINLAN- } \\
\text { DIA (pre- } \\
\text { universita- } \\
\text { rios) }\end{array}$ & $\begin{array}{l}\text { ESPAÑA/ } \\
\text { SALA- } \\
\text { MANCA }\end{array}$ & $\begin{array}{l}\text { ESPAÑA/ } \\
\text { MADRID }\end{array}$ & $\begin{array}{l}\text { TURQUÍA } \\
\text { (preuni- } \\
\text { versita- } \\
\text { rios) }\end{array}$ & $\begin{array}{l}\text { CHINA } \\
\text { (universi- } \\
\text { tarios) }\end{array}$ \\
\hline $\begin{array}{l}\text { pantalón } \\
0,70\end{array}$ & $\begin{array}{l}\text { pantalón } \\
0,75\end{array}$ & $\begin{array}{l}\text { zapato } \\
0,56\end{array}$ & $\begin{array}{l}\text { pantalón } \\
0,74\end{array}$ & $\begin{array}{l}\text { pantalón } \\
0,70\end{array}$ & $\begin{array}{l}\text { camisa } \\
0,61\end{array}$ & $\begin{array}{l}\text { pantalón } \\
0,65\end{array}$ \\
\hline $\begin{array}{l}\text { camisa } \\
0,63\end{array}$ & camisa & $\begin{array}{l}\text { pantalón } \\
0,69\end{array}$ & $\begin{array}{l}\text { camiseta } \\
0,41\end{array}$ & $\begin{array}{l}\text { camisa } \\
0,62\end{array}$ & $\begin{array}{l}\text { pantalón } \\
0,58\end{array}$ & $\begin{array}{l}\text { camisa } \\
0,57\end{array}$ \\
\hline $\begin{array}{l}\text { zapato } \\
0,42\end{array}$ & falda & $\begin{array}{l}\text { vaquero } \\
0,56\end{array}$ & $\begin{array}{l}\text { camisa } \\
0,36\end{array}$ & $\begin{array}{l}\text { camiseta } \\
0,48\end{array}$ & $\begin{array}{l}\text { zapato } \\
0,46\end{array}$ & $\begin{array}{l}\text { zapato } \\
0,54\end{array}$ \\
\hline
\end{tabular}

Cuadro 4: Las palabras más disponibles de La ropa en ELE 


\subsubsection{La casa}

Cocina es la palabra más disponible en todos los listados presentados en el campo semántico La casa aunque en ELE tiene el ID un poco más bajo que en ELM. Las palabras (cuarto de) baño y salón son también las más disponibles en ELM, y solo (cuarto de) baño en ELE. En ELE hay una uniformidad total en la palabra más disponible, cocina, pero hay menos convergencia en la segunda y la tercera palabra más disponible.

\begin{tabular}{|l|l|l|l|l|}
\hline PUERTO RICO & ASTURIAS & CÁDIZ & VALENCIA & MELILLA \\
\hline cocina & cocina & cocina & cocina & cocina \\
0,65 & 0,84 & 0,77 & 0,70 & 0,74 \\
\hline baño & cuarto de baño & salón & comedor & salón \\
0,59 & 0,74 & 0,72 & 0,65 & 0,66 \\
\hline sala & salón & cuarto de baño & cuarto de baño & (cuarto de) baño \\
0,58 & 0,71 & 0,67 & 0,58 & 0,63 \\
\hline
\end{tabular}

Cuadro 5: Las palabras más disponibles de La casa en ELM

\begin{tabular}{|c|c|c|c|c|c|}
\hline $\begin{array}{l}\text { ESLOVENIA } \\
\text { (preuniversi- } \\
\text { tarios) }\end{array}$ & $\begin{array}{l}\text { ESLOVENIA } \\
\text { (universita- } \\
\text { rios) }\end{array}$ & $\begin{array}{l}\text { FINLANDIA } \\
\text { (preuniversi- } \\
\text { tarios) }\end{array}$ & $\begin{array}{l}\text { ESPAÑA/ } \\
\text { SALAMAN- } \\
\text { CA }\end{array}$ & $\begin{array}{l}\text { TURQUÍA } \\
\text { (preuniversi- } \\
\text { tarios) }\end{array}$ & $\begin{array}{l}\text { CHINA } \\
\text { (universita- } \\
\text { rios) }\end{array}$ \\
\hline $\begin{array}{l}\text { cocina } \\
0,40\end{array}$ & $\begin{array}{l}\text { cocina } \\
0,64\end{array}$ & $\begin{array}{l}\text { cocina } \\
0,67\end{array}$ & $\begin{array}{l}\text { cocina } \\
0,67\end{array}$ & $\begin{array}{l}\text { cocina } \\
0,66\end{array}$ & $\begin{array}{l}\text { cocina } \\
0,53\end{array}$ \\
\hline $\begin{array}{l}\text { cama } \\
0,33\end{array}$ & $\begin{array}{l}\text { baño } \\
0,35\end{array}$ & $\begin{array}{l}\text { dormitorio } \\
0,47\end{array}$ & $\begin{array}{l}\text { baño } \\
0,51\end{array}$ & $\begin{array}{l}\text { dormitorio } \\
0,58\end{array}$ & $\begin{array}{l}\text { baño } \\
0,50\end{array}$ \\
\hline $\begin{array}{l}\text { mesa } \\
0,30\end{array}$ & $\begin{array}{l}\text { cama } \\
0,35\end{array}$ & $\begin{array}{l}\text { cuarto de } \\
\text { baño } 0,26\end{array}$ & $\begin{array}{l}\text { habitación } \\
0,44\end{array}$ & $\begin{array}{l}\text { salón } \\
0,54\end{array}$ & $\begin{array}{l}\text { mesa } \\
0,48\end{array}$ \\
\hline
\end{tabular}

Cuadro 6: Las palabras más disponibles de La casa en ELE

\subsubsection{Alimentos y bebidas}

En la categoría semántica Alimentos y bebidas la gran mayoría de los léxicos más disponibles presenta las bebidas y en pocos casos la comida: en ELM agua y coca-cola son los más disponibles, pero también vino, jugo, fanta y gïisqui. En ELE los más disponibles son agua y cerveza pero también leche, zumo y vino. En general, hay menos convergencia en esta categoría semántica y pocas palabras con un ID superior a 0,5 . 


\begin{tabular}{|l|l|l|l|l|}
\hline PUERTO RICO & ASTURIAS & CÁDIZ & VALENCIA & MELILLA \\
\hline arroz 0,84 & agua 0,59 & coca-cola 0,60 & agua 0,51 & coca-cola 0,76 \\
\hline habichuela 0,69 & coca-cola 0,50 & agua 0,42 & vino 0,33 & agua 0,47 \\
\hline jugo 0,41 & vino 0,37 & güísqui 0,38 & tomate 0,30 & fanta 0,43 \\
\hline
\end{tabular}

Cuadro 7: Las palabras más disponibles de Alimentos y bebidas en ELM

\begin{tabular}{|c|c|c|c|c|c|c|}
\hline $\begin{array}{l}\text { ESLOVE- } \\
\text { NIA } \\
\text { (preuni- } \\
\text { versita- } \\
\text { rios) }\end{array}$ & $\begin{array}{l}\text { ESLOVE- } \\
\text { NIA } \\
\text { (universi- } \\
\text { tarios) }\end{array}$ & $\begin{array}{l}\text { FINLAN- } \\
\text { DIA } \\
\text { (preuni- } \\
\text { versita- } \\
\text { rios) }\end{array}$ & $\begin{array}{l}\text { ESPAÑA/ } \\
\text { SALA- } \\
\text { MANCA }\end{array}$ & $\begin{array}{l}\text { ESPAÑA/ } \\
\text { MADRID }\end{array}$ & $\begin{array}{l}\text { TURQUÍA } \\
\text { (preuni- } \\
\text { versitarios) }\end{array}$ & $\begin{array}{l}\text { CHINA } \\
\text { (universi- } \\
\text { tarios) }\end{array}$ \\
\hline $\begin{array}{l}\text { agua } \\
0,49\end{array}$ & $\begin{array}{l}\text { agua } \\
0,45\end{array}$ & $\begin{array}{l}\text { leche } \\
0,54\end{array}$ & $\begin{array}{l}\text { agua } \\
0,50\end{array}$ & $\begin{array}{l}\text { cerveza } \\
0,55\end{array}$ & $\begin{array}{l}\text { agua } \\
0,52\end{array}$ & $\begin{array}{l}\text { arroz } \\
0,45\end{array}$ \\
\hline $\begin{array}{l}\text { cerveza } \\
0,41\end{array}$ & $\begin{array}{l}\text { zumo } \\
0,39\end{array}$ & $\begin{array}{l}\text { agua } \\
0,46\end{array}$ & $\begin{array}{l}\text { zumo } \\
0,49\end{array}$ & $\begin{array}{l}\text { agua } \\
0,44\end{array}$ & $\begin{array}{l}\text { leche } \\
0,45\end{array}$ & $\begin{array}{l}\text { carne } \\
0,49\end{array}$ \\
\hline $\begin{array}{l}\text { paella } \\
0,35\end{array}$ & $\begin{array}{l}\text { vino } \\
0,34\end{array}$ & $\begin{array}{l}\text { cerveza } \\
0,44\end{array}$ & $\begin{array}{l}\text { carne } \\
0,45\end{array}$ & $\begin{array}{l}\text { pan } \\
0,36\end{array}$ & $\begin{array}{l}\text { manzana } \\
0,32\end{array}$ & $\begin{array}{l}\text { vino } \\
0,42\end{array}$ \\
\hline
\end{tabular}

Cuadro 8: Las palabras más disponibles de Alimentos y bebidas en ELE

\subsubsection{La ciudad}

En la categoría abierta de La ciudad, cuatro de los cinco listados en ELM coinciden en la palabra más disponible que es cocbe con un ID bastante alto (entre 0,58 y o,71), mientras que en ELE coinciden sobre todo coche y calle, aparte de diferentes palabras de alto grado de disponibilidad que no coinciden entre los listados y cuyos ID son bajos.

\begin{tabular}{|l|l|l|l|l|}
\hline PUERTO RICO & ASTURIAS & CÁDIZ & VALENCIA & MELILLA \\
\hline tienda 0,47 & coche 0,64 & coche 0,60 & coche 0,71 & coche 0,58 \\
\hline edificio 0,40 & edificio 0,50 & calle 0,40 & moto(cicleta) 0,42 & carretera 0,45 \\
\hline carro 0,35 & parque 0,42 & casa 0,34 & (auto)bús 0,39 & edificio 0,37 \\
\hline
\end{tabular}

Cuadro 9: Las palabras más disponibles de La ciudad en ELM 


\begin{tabular}{|c|c|c|c|c|c|c|}
\hline $\begin{array}{l}\text { ESLOVE- } \\
\text { NIA } \\
\text { (preuni- } \\
\text { versita- } \\
\text { rios) }\end{array}$ & $\begin{array}{l}\text { ESLOVE- } \\
\text { NIA } \\
\text { (universi- } \\
\text { tarios) }\end{array}$ & $\begin{array}{l}\text { FINLAN- } \\
\text { DIA } \\
\text { (preuni- } \\
\text { versita- } \\
\text { rios) }\end{array}$ & $\begin{array}{l}\text { ESPAÑA/ } \\
\text { SALA- } \\
\text { MANCA }\end{array}$ & $\begin{array}{l}\text { ESPAÑA/ } \\
\text { MADRID }\end{array}$ & $\begin{array}{l}\text { TURQUÍA } \\
\text { (preuni- } \\
\text { versitarios) }\end{array}$ & $\begin{array}{l}\text { CHINA } \\
\text { (universi- } \\
\text { tarios) }\end{array}$ \\
\hline $\begin{array}{l}\text { calle } \\
0,47\end{array}$ & $\begin{array}{l}\text { coche } \\
0,54\end{array}$ & $\begin{array}{l}\text { calle } \\
0,51\end{array}$ & $\begin{array}{l}\text { calle } \\
0,69\end{array}$ & $\begin{array}{l}\text { coche } \\
0,53\end{array}$ & $\begin{array}{l}\text { cine } \\
0,24\end{array}$ & $\begin{array}{l}\text { edificio } \\
0,34\end{array}$ \\
\hline $\begin{array}{l}\text { coche } \\
0,46\end{array}$ & $\begin{array}{l}\text { gente } \\
0,44\end{array}$ & $\begin{array}{l}\text { casa } \\
0,39\end{array}$ & $\begin{array}{l}\text { coche } \\
0,43\end{array}$ & $\begin{array}{l}\text { calle } \\
0,43\end{array}$ & $\begin{array}{l}\text { café } \\
0,21\end{array}$ & $\begin{array}{l}\text { coche } \\
0,33\end{array}$ \\
\hline $\begin{array}{l}\text { casa } \\
0,37\end{array}$ & $\begin{array}{l}\text { calle } \\
0,43\end{array}$ & $\begin{array}{l}\text { coche } \\
0,32\end{array}$ & $\begin{array}{l}\text { tienda } \\
0,40\end{array}$ & $\begin{array}{l}\text { metro } \\
0,41\end{array}$ & $\begin{array}{l}\text { restaurante } \\
0,17\end{array}$ & $\begin{array}{l}\text { calle } \\
0,25\end{array}$ \\
\hline
\end{tabular}

Cuadro 10: Las palabras más disponibles de La ciudad en ELE

\subsubsection{El campo}

Otra categoría semántica abierta es El campo, donde prevalece el prototipo árbol en todos los léxicos disponibles en ELM pero los ID van de 0,40 a o,69. Hay también convergencia en la palabra flor y bierba pero con un índice por debajo de 0,4. Todos los informantes extranjeros de ELE comparten el prototipo animal, y la mayoría también comparte árbol (en tres de cinco grupos es la palabra más disponible) pero los ID varían mucho.

\begin{tabular}{|l|l|l|l|l|}
\hline PUERTO RICO & ASTURIAS & CÁDIZ & VALENCIA & MELILLA \\
\hline árbol 0,56 & árbol 0,50 & árbol 0,69 & árbol 0,39 & árbol 0,68 \\
\hline río 0,46 & vaca 0,43 & flor 0,38 & pino 0,37 & flor 0,36 \\
\hline animal 0,33 & flor 0,37 & hierba 0,35 & hierba 0,36 & hierba 0,31 \\
\hline
\end{tabular}

Cuadro 11: Las palabras más disponibles de El campo en ELM

\begin{tabular}{|l|l|l|l|l|}
\hline $\begin{array}{l}\text { ESLOVENIA } \\
\text { (preuniversita- } \\
\text { rios) }\end{array}$ & $\begin{array}{l}\text { ESLOVENIA } \\
\text { (universitarios) }\end{array}$ & $\begin{array}{l}\text { FINLANDIA } \\
\text { (preuniversita- } \\
\text { rios) }\end{array}$ & $\begin{array}{l}\text { ESPAÑA/ } \\
\text { SALAMANCA }\end{array}$ & $\begin{array}{l}\text { TURQUÍA } \\
\text { (preuniversita- } \\
\text { rios) }\end{array}$ \\
\hline vaca 0,52 & animal 0,48 & árbol 0,31 & árbol 0,76 & árbol 0,08 \\
\hline animal 0,43 & naturaleza 0,38 & bosque 0,27 & animal 0,56 & animal 0,05 \\
\hline naturaleza 0,32 & árbol 0,35 & animal 0,25 & río 0,39 & montaña 0,04 \\
\hline
\end{tabular}

Cuadro 12: Las palabras más disponibles de El campo en ELE 


\subsubsection{Medios de transporte}

En Medios de transporte, que es una categoría más cerrada, cocbe es el prototipo universal (con la expresión carro en Puerto Rico), menos en los informantes chinos, para los que la asociación más frecuente es autobús. Los índices para cocbe son extremadamente altos, entre 0,71 y o,85. En ELM también hay mucha convergencia en avión, autobús y moto, y en ELE autobús y tren. En todos los casos se trata de índices muy altos.

\begin{tabular}{|l|l|l|l|l|}
\hline PUERTO RICO & ASTURIAS & CÁDIZ & VALENCIA & MELILLA \\
\hline carro 0,73 & coche 0,85 & coche 0,83 & coche 0,76 & coche 0,83 \\
\hline avión 0,65 & avión 0,66 & (auto)bús 0,71 & avión 0,68 & (auto)bús 0,63 \\
\hline bicicleta 0,64 & (auto)bús 0,66 & $\begin{array}{l}\text { moto(cicleta) } \\
0,69\end{array}$ & $\begin{array}{l}\text { moto(cicleta) } \\
0,61\end{array}$ & avión 0,62 \\
\hline
\end{tabular}

Cuadro 13: Las palabras más disponibles de Medios de transporte en ELM

\begin{tabular}{|c|c|c|c|c|c|}
\hline $\begin{array}{l}\text { ESLOVENIA } \\
\text { (preuniversi- } \\
\text { tarios) }\end{array}$ & $\begin{array}{l}\text { ESLOVENIA } \\
\text { (universita- } \\
\text { rios) }\end{array}$ & $\begin{array}{l}\text { FINLANDIA } \\
\text { (preuniversi- } \\
\text { tarios) }\end{array}$ & $\begin{array}{l}\text { ESPAÑA/ } \\
\text { SALAMAN- } \\
\text { CA }\end{array}$ & $\begin{array}{l}\text { TURQUÍA } \\
\text { (preuniversi- } \\
\text { tarios) }\end{array}$ & $\begin{array}{l}\text { CHINA } \\
\text { (universita- } \\
\text { rios) }\end{array}$ \\
\hline $\begin{array}{l}\text { coche } \\
0,78\end{array}$ & $\begin{array}{l}\text { coche } \\
0,84\end{array}$ & $\begin{array}{l}\text { coche } \\
0,81\end{array}$ & $\begin{array}{l}\text { coche } \\
0,85\end{array}$ & $\begin{array}{l}\text { coche } \\
0,71\end{array}$ & $\begin{array}{l}\text { (auto)bús } \\
0,73\end{array}$ \\
\hline $\begin{array}{l}\text { bici(cleta) } \\
0,70\end{array}$ & $\begin{array}{l}\text { (auto)bús } \\
0,78\end{array}$ & $\begin{array}{l}\text { (auto)bús } \\
0,70\end{array}$ & $\begin{array}{l}\text { (auto)bús } \\
0,79\end{array}$ & $\begin{array}{l}\text { autobús } \\
0,65\end{array}$ & $\begin{array}{l}\text { coche } \\
0,70\end{array}$ \\
\hline $\begin{array}{l}\text { (auto)bús } \\
0,65\end{array}$ & $\begin{array}{l}\text { tren } \\
0,68\end{array}$ & $\begin{array}{l}\text { tren } \\
0,69\end{array}$ & $\begin{array}{l}\text { tren } \\
0,78\end{array}$ & $\begin{array}{l}\text { metro } \\
0,53\end{array}$ & $\begin{array}{l}\text { tren } \\
0,67\end{array}$ \\
\hline
\end{tabular}

Cuadro 14: Las palabras más disponibles de Medios de transporte en ELE

\subsubsection{Animales}

A pesar de las diferentes lenguas maternas y culturas de los informantes comparados, los prototipos universales en el campo semántico Animales son, sin duda, perro y gato, en todas las listas en este orden; perro como la primera y gato como la segunda asociación y siempre con índices muy altos.

\begin{tabular}{|l|l|l|l|l|}
\hline PUERTO RICO & ASTURIAS & CÁDIZ & VALENCIA & MELILLA \\
\hline perro 0,84 & perro 0,85 & perro 0,82 & perro 0,77 & perro 0,88 \\
\hline gato 0,76 & gato 0,80 & gato 0,77 & gato 0,75 & gato 0,79 \\
\hline caballo 0,59 & vaca 0,54 & león 0,45 & león 0,55 & león 0,40 \\
\hline
\end{tabular}

Cuadro 15: Las palabras más disponibles de Animales en ELM 


\begin{tabular}{|l|l|l|l|l|l|}
\hline $\begin{array}{l}\text { ESLOVENIA } \\
\text { (preuniversi- } \\
\text { tarios) }\end{array}$ & $\begin{array}{l}\text { ESLOVENIA } \\
\text { (universita- } \\
\text { rios) }\end{array}$ & $\begin{array}{l}\text { FINLANDIA } \\
\text { (preuniversi- } \\
\text { tarios) }\end{array}$ & $\begin{array}{l}\text { ESPAÑA/ } \\
\text { SALAMAN- } \\
\text { CA }\end{array}$ & $\begin{array}{l}\text { TURQUÍA } \\
\text { (preuniversi- } \\
\text { tarios) }\end{array}$ & $\begin{array}{l}\text { CHINA } \\
\text { (universita- } \\
\text { rios) }\end{array}$ \\
\hline perro 0,79 & perro 0,80 & perro 0,82 & perro 0,84 & perro 0,79 & perro 0,82 \\
\hline gato 0,78 & gato 0,77 & gato 0,67 & gato 0,82 & gato 0,75 & gato 0,76 \\
\hline vaca 0,53 & vaca 0,58 & caballo 0,47 & vaca 0,54 & caballo 0,37 & tigre 0,41 \\
\hline
\end{tabular}

Cuadro 16: Las palabras más disponibles de Animales en ELE

\subsubsection{Profesiones y oficios}

Hay muchísima unanimidad también en el campo de Profesiones y oficios con los prototipos de profesor / maestro como primera asociación, médico / doctor como segunda y abogado como tercera. Solo profesor / maestro tiene un índice superior a 0,50 , los demás índices son más bajos. Cabe añadir que las pruebas asociativas se hacen normalmente en el ámbito escolar, lo que puede provocar la asociación de profesor. De todas estas palabras podríamos destacar las más disponibles y prototípicas a la vez, las que muestran un ID más alto. En este sentido destacan perro y gato, coche, profesor, pantalón, camisa, ojo, cabeza y cocina.

\begin{tabular}{|l|l|l|l|l|}
\hline PUERTO RICO & ASTURIAS & CÁDIZ & VALENCIA & MELILLA \\
\hline maestro 0,52 & profesor 0,67 & profesor 0,60 & profesor 0,50 & profesor 0,61 \\
\hline doctor 0,47 & médico 0,44 & médico 0,40 & médico 0,38 & médico 0,41 \\
\hline secretaria 0,45 & abogado 0,34 & abogado 0,30 & abogado 0,34 & policía 0,40 \\
\hline
\end{tabular}

Cuadro 17: Las palabras más disponibles de Profesiones y oficios en ELM

\begin{tabular}{|c|c|c|c|c|c|}
\hline $\begin{array}{l}\text { ESLOVENIA } \\
\text { (preuniversi- } \\
\text { tarios) }\end{array}$ & $\begin{array}{l}\text { ESLOVENIA } \\
\text { (universita- } \\
\text { rios) }\end{array}$ & $\begin{array}{l}\text { FINLANDIA } \\
\text { (preuniversi- } \\
\text { tarios) }\end{array}$ & $\begin{array}{l}\text { ESPAÑA/ } \\
\text { SALAMAN- } \\
\text { CA }\end{array}$ & $\begin{array}{l}\text { TURQUÍA } \\
\text { (preuniversi- } \\
\text { tarios) }\end{array}$ & $\begin{array}{l}\text { CHINA } \\
\text { (universita- } \\
\text { rios) }\end{array}$ \\
\hline $\begin{array}{l}\text { profesor } \\
0,65\end{array}$ & $\begin{array}{l}\text { profesor } \\
0,82\end{array}$ & $\begin{array}{l}\text { profesor } \\
0,80\end{array}$ & $\begin{array}{l}\text { profesor } \\
0,81\end{array}$ & $\begin{array}{l}\text { profesor } \\
0,56\end{array}$ & $\begin{array}{l}\text { profe(sor) } \\
0,84\end{array}$ \\
\hline $\begin{array}{l}\text { médico } \\
0,35\end{array}$ & $\begin{array}{l}\text { médico } \\
0,47\end{array}$ & $\begin{array}{l}\text { médico } \\
0,41\end{array}$ & $\begin{array}{l}\text { médico } \\
0,38\end{array}$ & $\begin{array}{l}\text { médico } \\
0,44\end{array}$ & $\begin{array}{l}\text { estudiante } \\
0,40\end{array}$ \\
\hline $\begin{array}{l}\text { actor } \\
0,31\end{array}$ & $\begin{array}{l}\text { abogado } \\
0,33\end{array}$ & $\begin{array}{l}\text { policía } \\
0,22\end{array}$ & $\begin{array}{l}\text { abogado } \\
0,31\end{array}$ & $\begin{array}{l}\text { abogado } \\
0,34\end{array}$ & $\begin{array}{l}\text { médico } \\
0,33\end{array}$ \\
\hline
\end{tabular}

Cuadro 18: Las palabras más disponibles de Profesiones y oficios en ELE 


\section{Conclusiones}

Comparando las palabras más disponibles en hablantes nativos y extranjeros de español, es decir, la parte colectiva de los inventarios, se ha demostrado un alto grado de similitud en las palabras prototípicas a pesar de las culturas y lenguas diferentes de los informantes. Además de funcionar estas palabras como las asociaciones primarias o prototipos, las palabras como perro, gato, cocbe, profesor, pantalón, camisa, ojo, cabeza, cocina (las palabras más disponibles y dotadas de un ID alto) y también baño, agua, árbol, animal, autobús, tren (las palabras más disponibles con un ID un poco más bajo) son las palabras con las cuales entramos en contacto en la vida diaria y que aprendimos durante nuestra infancia. Con esto se confirman las características de los prototipos según Kleiber (1995) y los tres factores que según Hernández Muñoz e Izura (2010: 2) predicen la disponibilidad.

El cotejo presentado en los cuadros ha confirmado también que «para las teorías asociacionistas de la psicolingüística son más relevantes las áreas temáticas que potencian un inventario más compacto o cerrado en la actualización de las unidades léxicas» (Gómez Molina y Gómez Devís, 2004: 280). Las categorías semánticas donde los índices de disponibilidad son los más altos son Las partes del cuerpo, La ropa, La casa, Medios de transporte y Animales y coinciden con las áreas semánticas con mayor cohesión semántica que citan los mismos autores (Ibid.).

Se ha confirmado también que la gran mayoría de los prototipos son de nivel de base según la teoría de los prototipos, menos, por ejemplo, el prototipo de animal en un campo semántico muy abierto: El campo.

Muchos lingüistas que han investigado el léxico disponible en ELE confirman que los informantes de diferentes niveles han mostrado gran convergencia en las palabras más disponibles, es decir, que a pesar de un conocimiento mayor o menor del español producen los mismos prototipos, lo que también se ha confirmado con el presente cotejo, en el que se comparan los léxicos de estudiantes preuniversitarios y universitarios. Así afirma Jing (2012: 12) que «también se observa un elevado grado de convergencia que se pone de manifiesto en los vocablos de la máxima disponibilidad léxica para los dieciséis centros de interés en los tres niveles de español sometidos a la prueba». Igualmente afirma también Carcedo González (2000: 132) sobre el cotejo de cuatro grupos de informantes (escolares y universitarios) que «la convergencia del vocabulario entre los grupos es especialmente visible en los primeros puestos de la lista»y «que todo ello nos habla de una gran similitud en el tipo de asociaciones que 
en los distintos estadios del aprendizaje establecen los sujetos finlandeses que componen la muestra» (2000: 134). Según Bartol Hernández (2010: 100) la tipicidad y la edad de adquisición son los factores responsables de la unidad del léxico, de las semejanzas que se establecen entre el léxico de diferentes zonas también en los sujetos alejados geográfica y culturalmente.

Finalmente podemos concluir que las encuestas de disponibilidad léxica realizadas tanto en lengua materna como extranjera ilustran claramente la categorización humana desde el punto de vista de los prototipos y confirman la universalidad del lenguaje. La disponibilidad léxica, por lo tanto, puede servir como una herramienta eficaz para investigar las asociaciones humanas.

\section{Bibliografía}

Aitchison, J. (1994): Words in the Mind: An Introduction to the Mental Lexicon. Oxford - New York: Basil Blackwell.

Bartol Hernández, J. A. (2010): «Disponibilidad léxica y selección del vocabulario». En: Rosa M. ${ }^{a}$ Castañer Martín y Vicente Lagüens Gracia (eds.), De moneda nunca usada. Estudios filológicos dedicados a José M. ${ }^{a}$ Enguita Utrilla, Institución «Fernándo el Católico»: Zaragoza, 85-107.

Carcedo González, A. (200o): Disponibilidad léxica en español como lengua extranjera: el caso finlandés (estudio del nivel preuniversitario y cotejo con tres fases de adquisición). Turku: Universidad de Turku.

Carcedo González, A. (2001): Léxico disponible de Asturias. Turku: Universidad de Turku.

Férnandez Smith, G., Rico Martín, A. $M^{\mathrm{a}}$, Molina García, $M^{\mathrm{a}}$ J., Jiménez

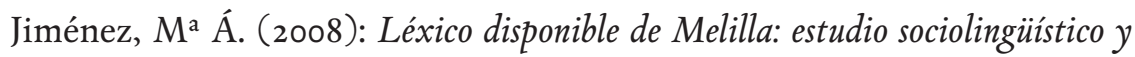
repertorios léxicos. Madrid: Arco Libros.

Gómez Molina, J. R., Gómez Devís, Ma B. (2004): La disponibilidad léxica de los estudiantes preuniversitarios valencianos. Estudio de estratificación sociolingüistica. Valencia: Universidad de Valencia.

González Fernández, J. (2013): «La disponibilidad léxica de los estudiantes turcos de español como lengua extranjera». En: MarcoELE, 16. [en línea]

González Martínez, A. (2002): La disponibilidad léxica de los alumnos preuniversitarios de la provincia de Cádiz. Cádiz: Universidad de Cádiz.

Hernández Muñoz, N., Izura, C. (2010): «¡Perro o musaraña? La recuperación léxica en las categorías semánticas». En: Ciencia cognitiva, 4:1, 1-3. [en línea] 
Hernández Muñoz, N., Izura, C., Tomé, C. (2014): «Cognitive Factors of Lexical Availability in a Second Language». En Rosa M. Jiménez Catalán (ed.), Lexical Availability in English and Spanish as a Second Language. Dordrecht, Heidelberg, London, New York: Springer, 169-186.

Jing, L. (2012): «El estudio de disponibilidad léxica de los estudiantes chinos de español como lengua extranjera». En: MarcoELE, 14. [en línea]

Kleiber, G. (1995): La semántica de los prototipos. Categoría y sentido léxico. Madrid: Visor.

Lakoff, G. (1987): Women, Fire, And Dangerous Things. Chicago: University Of Chicago Press.

López Rivero, E. (2008): Estudio de disponibilidad léxica en 43 estudiantes de ELE. Madrid: Universidad Antonio de Nebrija. Memoria del máster en enseñanza del español como lengua extranjera. [en línea]

Luque Durán, J. (2004): Aspectos universales y particulares del léxico de las lenguas del mundo. Granada: Impredisur.

López Morales, H. (1999): Léxico disponible de Puerto Rico. Madrid: Arco Libros.

Martos Ramos, J. J. (2010): «La búsqueda del prototipo: reflexiones sobre los parametros de categorizaron». En: Revista de Filologia Alemana, 18, 247259. [en línea]

Pinker, S. (1995): El instinto del lenguaje. Cómo crea el lenguaje la mente. Madrid: Alianza Editorial.

Pinker, S. (2010): Jezikovni nagon. Kako um ustvarja jezik. Ljubljana: Modrijan.

Samper Hernández, M. (2002): Disponibilidad léxica en alumnos de español como lengua extranjera. Málaga: ASELE, Colección Monografías, 4.

Šifrar Kalan, M. (2011): Leksikalna razpoložljivost v španščini kot tujem jeziku. Ljubljana: Universidad de Ljubljana. [tesis doctoral]

Šifrar Kalan, M. (2012): «Análisis comparativo de la disponibilidad léxica en español como lengua extranjera (ELE) y lengua materna (ELM)». En: MarcoELE, Revista de didáctica ELE, 15, 1-19. [en línea]

Šifrar Kalan, M. (2013): «Slovene Students» Lexical Availability in English and Spanish». En: Rosa M. Jiménez Catalán, (ed.), Lexical Availability in English and Spanish as a Second Language. Dordrecht, Heidelberg, London, New York: Springer, 125-138.

Taylor, J. R. (1995) Linguistic Categorization. Prototypes in Linguistic Theory. Oxford: Clarendon Press. 
Marjana Šifrar Kalan

University of Ljubljana

\section{The Universality of Semantic Prototypes in Spanish Lexical Availability}

Keywords: semantic prototypes, lexical availability, prototype theory, most available words, Spanish as a foreign language

The article presents the words with highest index of availability on the basis of semantic fluency tests. The conceptual stability of highly available words in various semantic categories enables them to be classified as semantic prototypes according to the theory of prototype. The aim of this article is to compare the semantic prototypes in nine semantic categories from different lexical availability studies: those carried out in Spanish as a mother tongue and Spanish as a foreign language (with Slovene, Finnish, Turkish, Chinese students and students of various other mother tongues who studied Spanish in Madrid and Salamanca). The informants who come from different countries and cultures and speak different first languages demonstrate that human beings share the same or similar categorization and universality of semantic prototypes. 
Marjana Šifrar Kalan

Univerza v Ljubljani

\section{Univerzalnost semantičnih prototipov $\mathbf{v}$ španskem razpoložljivem besedišču}

Ključne besede: semantični prototipi, leksikalna razpoložljivost, teorija prototipov, najbolj razpoložljive besede, španščina kot tuji jezik

Na podlagi rezultatov razpoložljivega besedišča, pridobljenega s testom semantične tekočnosti, članek predstavi besede z najvišjim indeksom razpoložljivosti. Pojmovna stabilnost teh besed iz različnih semantičnih polj nam omogoča, da jih na podlagi teorije prototipov uvrstimo med semantične prototipe. Namen članka je primerjati semantične prototipe $\mathrm{v}$ devetih semantičnih poljih različnih študij leksikalne razpoložljivosti: tistih, ki proučujejo španščino kot materni jezik, in tistih, ki jo proučujejo kot tuji jezik (med slovenskimi, finskimi, turškimi in kitajskimi študenti ter študenti različnih maternih jezikov, ki so se španščine učili v Salamanki in Madridu). Informanti, ki izvirajo iz različnih držav in kultur ter govorijo zelo različne materne jezike, dokazujejo, da si človeška bitja delijo enako ali podobno kategorizacijo in univerzalnost semantičnih prototipov. 